CLINICAL STUDY

\title{
Five novel mutations in the SCNN1A gene causing autosomal recessive pseudohypoaldosteronism type 1
}

\author{
Maik Welzel, Leyla Akin ${ }^{8}$, Anja Büscher ${ }^{6}$, Tülay Güran ${ }^{10}$, Berthold P Hauffa ${ }^{5}$, Wolfgang Högler ${ }^{7}$, Julia Leonards ${ }^{9}$, \\ Beate Karges $^{1,2}$, Heiner Kentrup ${ }^{1}$, Birgul Kirel ${ }^{4}$, Emine Esin Yalinbas Senses ${ }^{3}$, Neslihan Tekin ${ }^{3}$, \\ Paul-Martin Holterhus and Felix G Riepe \\ Division of Pediatric Endocrinology and Diabetes, Department of Pediatrics, University Hospital Schleswig-Holstein, Christian-Albrechts University, \\ Schwanenweg 20, D-24105 Kiel, Germany, ${ }^{1}$ Department of Pediatrics, Bethlehem Krankenhaus, RWTH Aachen University, 52222 Stolberg, Germany, \\ ${ }^{2}$ Division of Endocrinology and Diabetes, RWTH Aachen University, Stolberg, Germany, ${ }^{3}$ Division of Neonatology and ${ }^{4}$ Division of Pediatric Endocrinology, \\ Faculty of Medicine, Osmangazi University, Eskisehir, Turkey, ${ }^{5}$ Division of Pediatric Endocrinology and Diabetes, Department of Pediatrics, University of \\ Essen, Essen, Germany, ${ }^{6}$ Division of Pediatric Nephrology, Department of Pediatrics, University of Essen, Essen, Germany, ${ }^{7}$ Department of Endocrinology \\ and Diabetes, Birmingham Children's Hospital, Birmingham, UK, ${ }^{8}$ Department of Pediatric Endocrinology, Faculty of Medicine, Erciyes University, \\ Kayseri, Turkey, ${ }^{9}$ St Marien-Hospital Robert-Koch-Straße 1, 53115 Bonn, Germany and ${ }^{10}$ Division of Pediatric Endocrinology and Diabetes, Marmara \\ University Hospital, 81190 Altunizade-Istanbul, Turkey
}

(Correspondence should be addressed to M Welzel; Email: m.welzel@pediatrics.uni-kiel.de)

\begin{abstract}
Background: Pseudohypoaldosteronism type 1 (PHA1) is a monogenic disease caused by mutations in the genes encoding the human mineralocorticoid receptor (MR) or the $\alpha$ (SCNN1A), $\beta$ (SCNN1B) or $\gamma(S C N N 1 G)$ subunit of the epithelial $\mathrm{Na}^{+}$channel (ENaC). While autosomal dominant mutation of the MR cause renal PHA1, autosomal recessive mutations of the ENaC lead to systemic PHA1. In the latter, affected children suffer from neonatal onset of multi-organ salt loss and often exhibit cystic fibrosis-like pulmonary symptoms.

Objective: We searched for underlying mutations in seven unrelated children with systemic PHA1, all offsprings of healthy consanguineous parents.

Methods and results: Amplification of the SCNN1A gene and sequencing of all 13 coding exons unraveled mutations in all of our patients. We found five novel homozygous mutations (c.587_588insC in two patients, c.1342_1343insTACA, c.742delG, c.189C>A, c.1361-2A $>$ G) and one known mutation (c. $1474 \mathrm{C}>\mathrm{T}$ ) leading to truncation of the $\alpha \mathrm{ENaC}$ protein. All parents were asymptomatic heterozygous carriers of the respective mutations, confirming the autosomal recessive mode of inheritance. Five out of seven patients exhibited pulmonary symptoms in the neonatal period. Conclusion: The $\alpha$ subunit is essential for ENaC function and mutations truncating the pore-forming part of the protein leading to systemic PHA1. Based on current knowledge, the pulmonary phenotype cannot be satisfactorily predicted.
\end{abstract}

European Journal of Endocrinology 168 707-715

\section{Introduction}

Pseudohypoaldosteronism type 1 (PHA1) is a rare disease caused by defective transepithelial sodium transport. Affected children develop life-threatening salt loss of neonatal onset, hyperkalemia, and acidosis. Typically, these children have elevated aldosterone levels due to end organ resistance to aldosterone. Accordingly, they are insensitive to mineralocorticoid treatment but respond to high doses of sodium supplementation and ion exchange resins. At least two clinical forms of PHA1 can be distinguished. The renal form (OMIM \# 177735) is characterized by salt loss exclusively through the kidneys. It is caused by autosomal dominant mutations in the human mineralocorticoid receptor (MR, NR3C2) gene (1). In most of the patients with the renal form of PHA1, sodium supplementation can be discontinued by the age of $1-3$, probably due to the maturation of the renal salt conservation abilities (2). However, one patient reported to be compound heterozygous for two mutations of the MR exhibited a clinical phenotype comparable to systemic PHA1. A cutaneous or pulmonary phenotype, though, was not observed (3). In contrast, in the systemic form of PHA1 (OMIM \# 264350), salt loss results not only from the kidneys but also from the colon, salivary glands, and sweat ducts. Affected patients require life-long salt supplements and medical assistance and some are prone to develop a cystic fibrosis-like pulmonary phenotype with recurrent infections (4). Although this is the predominant phenotype, partially inactivating mutations may lead to large inter-individual differences. Dirlewanger reported on a term-born who had only slightly 
decreased sodium levels while its sibling developed a full-blown salt-losing syndrome (5).

The molecular genetic causes for systemic PHA1 are autosomal recessive mutations in genes encoding the amiloride-sensitive epithelial $\mathrm{Na}^{+}$channel (ENaC) (6). The $\mathrm{ENaC}$ is a heterotrimer formed by the three subunits, $\alpha, \beta$, and $\gamma(7,8,9,10)$. All subunits are thought to have a comparable three-dimensional structure containing two transmembrane segments, intracellular $\mathrm{N}$ - and C-termini, and a large extracellular loop (11). Additionally, the C-terminus contains a proline-rich domain involved in interaction with regulatory proteins. The subunits are encoded by the SCNN1A (chromosome 12p13.31), SCNN1B (chromosome 16p12.1), and SCNN1G (chromosome 16p12.1) genes. In systemic PHA1, mutations in these three genes lead to truncated or abnormal nonfunctional proteins $(6,12,13,14)$. Interestingly, mutations in the proline-rich domain cause the counterpart of systemic PHA1, Liddle's disease (15). In this paper, we present five novel and one previously reported nonsense mutation found in seven unrelated children with systemic PHA1.

\section{Materials and methods}

\section{Patient characteristics}

All children experienced dehydration with life-threatening renal sodium-loss and hyperkalemia during their first 2 weeks of life. Markedly elevated levels of aldosterone suggested PHA1 and unresponsiveness to fludrocortisone treatment further pinned down the diagnosis toward systemic PHA1. Biochemical results are summarized in Table 1. Individual information on their history and medical management is given below.

Patient 1 (c.587_588insC) This boy was born after uneventful pregnancy to healthy consanguineous parents (first cousins) of Turkish origin. He presented on the 10th day of life with failure to thrive, dehydration, hyponatremia, and hyperkalemia. Initially, he received i.v. sodium supplementation, ion exchange resins, and glucose/ insulin infusion to lower potassium levels. After discharge, he was put on oral sodium supplementation, but he suffered from recurrent salt-losing crises throughout childhood and exhibited a cystic fibrosis-like phenotype with bronchiectasis. His mental development is normal. At the age of 17 years, he reached normal height but suffered from obesity. Oral daily sodium supplementation is $28 \mathrm{~g}(\sim 11.5 \mathrm{mmol} / \mathrm{kg}$ per day $)$.

Patient 2 (c.1342_1343instACA) This girl was born after uneventful pregnancy to healthy consanguineous parents (second cousins) of Turkish origin. She was admitted to hospital because of recurrent vomiting, dehydration, and somnolence on the 8th day

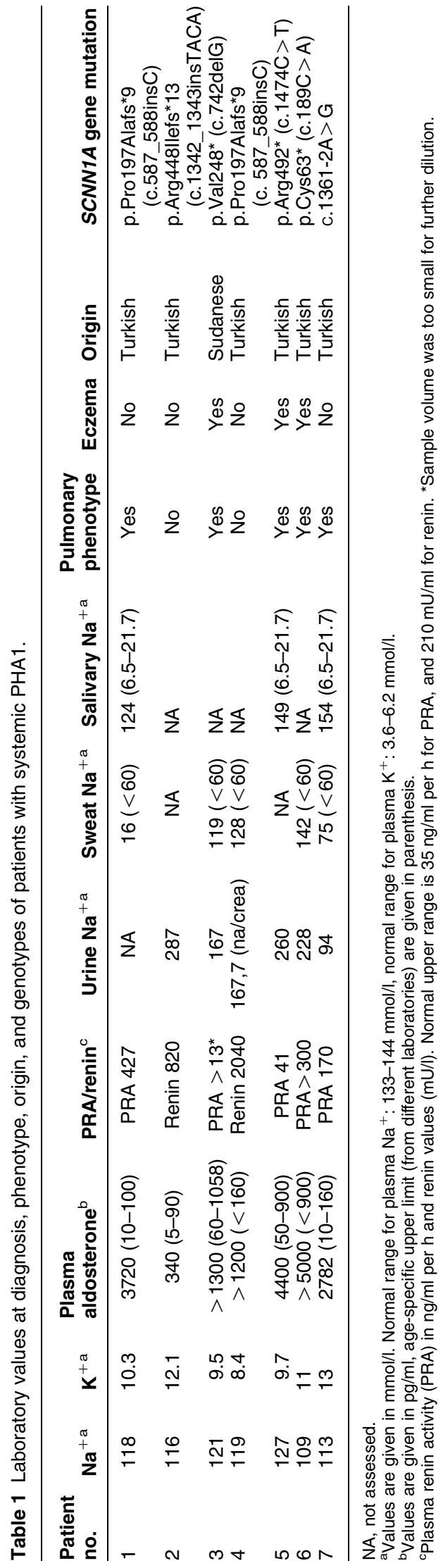

www.eje-online.org 
of life. Initial work-up showed markedly low sodium levels, elevated potassium levels, and hypotension. Steroid 21-hydroxylase deficiency, the commonest form of congenital adrenal hyperplasia (CAH), was ruled out by normal 17-OH progesterone levels. She received a combination therapy of ion exchange resins, glucose/insulin, hemodialysis, and i.v. sodium supplementation.

At the age of $11^{1 / 2}$ years, mental development is normal and the patient is of normal height and weight. She does not show any pulmonary symptoms, but saltlosing crises occur approximately once a year during intercurrent infections.

Patient 3 (c.742delG) Patient 3 was born after uneventful pregnancy to consanguineous parents (first cousins) of Sudanese (African) origin. The mother suffered from bronchial asthma and previously had one stillbirth and one miscarriage. On the 11th day of life, he was admitted to hospital with dehydration, acidosis ( $\mathrm{pH} 7.24$ ), ventricular tachycardia, and respiratory distress. A short cardio pulmomary resuscitation (CPR) cycle was performed when he was without vital signs. Renal salt loss was corrected by i.v. sodium supplementation; hyperkalemia by dextrose infusion and ion exchange resins. He subsequently developed septic arthritis of the left hip requiring arthrotomy and washout at 3 weeks of life. Throughout his infancy to date, he had recurrent mild but prolonged respiratory tract infections and dry and itchy eczema-like skin lesions.

At the age of 21 months, the patient showed normal development but struggled to gain weight (height SDS -1.87 and weight SDS -2.15 (16)). He is currently on an oral $\mathrm{NaCl}$ dose of $19 \mathrm{mmol} / \mathrm{kg}$ body weight and polystyrene sulphonate resins $(0.4 \mathrm{~g} / \mathrm{kg})$ daily. Sodium levels are borderline low as his parents are reluctant to try application by NG tube, but he takes the concentrated supplements orally. So far, no salt-losing crises have occurred.

Patient 4 (c.587_588insC) This girl was born by cesarean section after uneventful pregnancy to consanguineous parents (first cousins) of Turkish origin. For the first 9 days of life, she was treated with antibiotics for suspected newborn sepsis. After discharge, she was readmitted to hospital on the 17 th day of life with apathy and dehydration. Hyponatremia and hyperkalemia were treated with i.v. sodium supplementation ( $\max 85 \mathrm{mmol} / \mathrm{kg}$ body weight), albuterol, and glucose/insulin infusion.

At the age of three, the patient showed normal psychomotor development. However, because of failure to thrive, a percutaneous gastrostomy was introduced. So far, she experienced two pulmonary infections. Sodium and potassium levels greatly varied using 20-28 mmol/kg body weight sodium daily. Height at the age of 3 10/12 years was $94.5 \mathrm{~cm}(-2.17$ SDS $)$ and weight was $14.2 \mathrm{~kg}$ (BMI SDS - 0.12 (17)).
Patient 5 (c.1474C $>$ T) Patient 5 was born after uneventful pregnancy to consanguineous parents (second cousins) of Turkish origin. The last fetal ultrasound scan reported 'fetal nephromegaly'. On the 9th day of life he presented hyperbilirubinemia. Initial evaluation revealed moderately low sodium and elevated potassium levels that subsequently worsened. I.v. sodium supplementation, and ion exchange resins were insufficient to control $\mathrm{Na} / \mathrm{K}$ homeostasis. Therefore, peritoneal dialysis was started.

At the age of 11 months, he had a cardiac arrest during an acute illness with salt-losing crisis. CPR was successful and at the age of 20 months, he is developing normally. His height was $82 \mathrm{~cm}(-0.7$ SDS $)$ and weight $10 \mathrm{~kg}(-1.2$ SDS (18)). He had recurrent salt-losing crises during acute illness, pulmonary infections, and mild rashes. $\mathrm{Na} / \mathrm{K}$ homeostasis is controlled using $34 \mathrm{mmol} / \mathrm{kg}$ sodium body weight daily.

Patient $6(\boldsymbol{c . 1 8 9 C}>\boldsymbol{A})$ Patient 6 was a boy born after uneventful pregnancy to consanguineous parents (second degree relatives) of Turkish origin. On day 2 he required phototherapy for hyperbilirubinemia. On day 6 he was transferred due to worsening of his general condition. Hyponatremia and hyperkalemia raised the suspicion of $\mathrm{CAH}$ and combined mineralocorticoid/ glucocorticoid treatment was started. Albuterol, glucose/ insulin, and bicarbonate were insufficient to lower potassium levels; thus, peritoneal dialysis was initiated and ion exchange resin was added to the treatment schedule. Treatment for $\mathrm{CAH}$ was unsuccessful and discontinued. Elevated urinary sodium and low potassium excretion in combination with increased plasma aldosterone levels led to the diagnosis of PHA1. Oral $\mathrm{Na}$ supplementation of $17 \mathrm{mmol} / \mathrm{kg}$ per day was required to maintain serum sodium concentrations. On follow-up, he had slight neuromotor developmental delay, recurrent pulmonary infections, and an atopic dermatitis-like rash. He died at 11 months of age due to severe electrolyte imbalance during sepsis.

Patient 7 (c.1361-2A $>$ G) Patient 7 was born after uneventful pregnancy to consanguineous parents (third cousins) of Turkish origin. On the 7th day of life she was admitted to hospital, dehydrated and lethargic. Blood pressure was $65 / 40 \mathrm{mmHg}, \mathrm{K}^{+}$ $13 \mathrm{mmol} / \mathrm{l}$, and $\mathrm{Na}^{+} 113 \mathrm{mmol} / \mathrm{l}$. Hyponatremia and hyperkalemia were treated with i.v. sodium supplementation (20-30 mmol/kg body weight), albuterol, glucose/insulin infusion, ion exchange resin, and a single session of peritoneal dialysis. After 21-OHD was ruled out by normal 17-OH progesterone levels, glucocorticoid replacement was stopped. High sweat and urinary sodium excretion suggested PHA1.

At the age of $8 \frac{1}{2}$ months, the patient shows normal somatic and neuromotor development. So far, she was hospitalized once for hyponatremic convulsion and four 


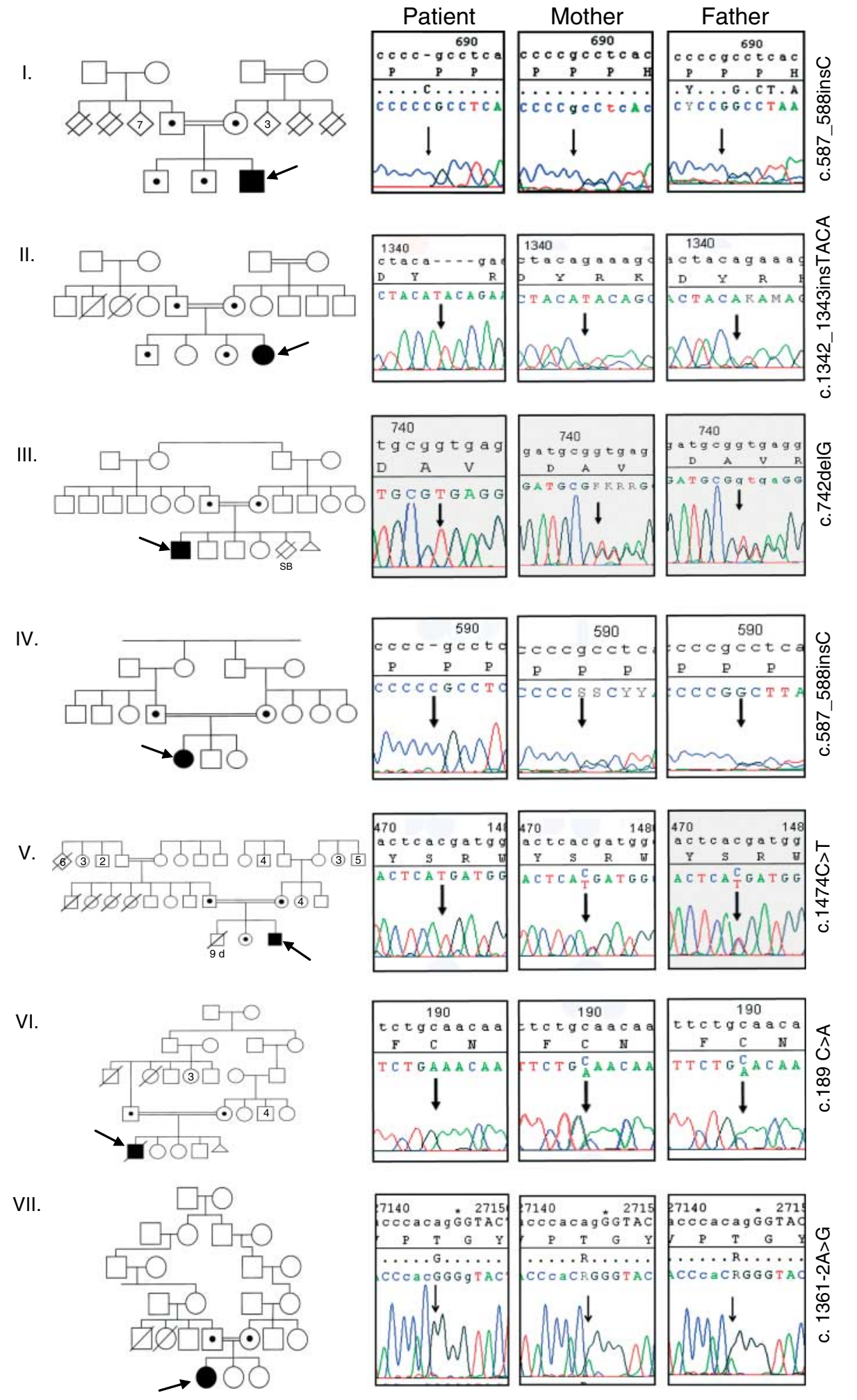


times for severe bronchiolitis attacks. $\mathrm{Na} / \mathrm{K}$ homeostasis is normal using $10 \mathrm{mmol} / \mathrm{kg}$ sodium body weight daily.

\section{Mutational analysis}

The study was approved by the local ethical review board. Blood samples for DNA analysis were obtained after informed consent from all patients and/or their parents. Preparation of genomic DNA from peripheral blood leukocytes followed a standard protocol. All 13 exon and the exon-intron boundaries of the SCNN1A gene were amplified by PCR using $\sim 500 \mathrm{ng}$ genomic DNA, $0.7 \mu \mathrm{l}$ Hot Start Taq, dNTPs, and buffers according to the manufacturer's protocol (Qiagen) as described previously (14). Primers are given in a Supplementary Table 1, see section on supplementary data given at the end of this article. Sequence variations were confirmed in an independent experiment. Mutants were designated according to the recommendations of the Nomenclature Working Group (19) based on the cDNA reference sequence NM_001038.5.

\section{Results}

\section{Mutational analysis}

Complete DNA sequencing of the SCNN1A gene revealed novel homozygous mutations in five patients.

Patients 1 and 4 carried an insertion of cytosine in exon 3 (c.587_588insC) causing a frame-shift with insertion of a premature stop codon at position 205 of the $\alpha \mathrm{ENaC}$ protein (p.P197A, H198S...S205X; p.Pro197Alafs*9). In patient 1 both the parents and the brothers were heterozygous carriers of the mutation (Fig. 1.I). Parents of patient 4 were also carriers, confirming the autosomal recessive mode of inheritance (Fig. 1.IV).

Patient 2 carried a homozygous insertion of TACA (c.1342_1343insTACA) in exon 10 leading to a frameshift and introducing a premature stop codon at position 460 (p.R448I...L460X; p.Arg448Ilefs*13) of the $\alpha \mathrm{ENaC}$ protein. Both parents were heterozygous carriers. A sister was ruled out as carrier (Fig. 1.II).

Mutational analysis in patient 3 revealed a deletion of guanine (c.742delG) in exon 4 . The mutation introduces a premature stop codon at residue 248 (p.Val248*) causing truncation of the $\alpha \mathrm{ENaC}$ protein. Both parents were carriers of the p.Val248* mutation (Fig. 1.III). The patient's siblings (two brothers and a sister) were not available for investigation.

In patient 5 , we found a homozygous missense mutation (c.1474C $>\mathrm{T}$ ) in exon 10, predicting an amino acid substitution of arginine by a stop codon (p.Arg492*). The mutation was found in the heterozygous form in both parents and the healthy sister (Fig. 1.V).

Mutational analysis in patient 6 unveiled a homozygous mutation in exon 2 (c.189C $>$ A). The mutation introduces a premature stop codon at residue 63 (p.Cys63*). Autosomal recessive inheritance was corroborated as both parents are carriers (Fig. 1.VI).

Patient 7 had a homozygous adenine to guanine substitution in the intron-exon boundary of intron 8/exon 9, changing the splice acceptor site from AG to GG (c.1361-2A>G). The consanguineous parents were heterozygous for this mutation (Fig. 1.VII).

\section{Discussion}

This study describes seven patients from apparently unrelated families. After the period of physiologic aldosterone resistance (20), each individual presented with life-threatening salt-loss within the second week of life. In most patients, $\mathrm{CAH}$ was initially suspected as it is the most common cause of neonatal onset of salt-losing crisis. Typically, none of the patients responded adequately to fludrocortisone treatment, ruling out $\mathrm{CAH}$. Unlike acute changes of salt homeostasis that might cause cardiac arrest or seizures, clinical signs of a progressive salt loss may be subtle in

Figure 1 Mutations in the SCNN1A gene in PHA1 kindreds. Pedigrees using the Standardized Human Pedigree Nomenclature (36) are shown on the left. Index patients are highlighted by an arrow. Filled symbols represent homozygosity, open symbols wild-type carriers. Carriers are marked with a central dot. Family members deceased during early infancy are shown as slashed symbols with the day of life shown below, if known. SB, stillbirth; triangles, miscarriages. The right panel shows the electropherograms of patients, mothers, and fathers (from left to right) by direct DNA sequencing as described in the Materials and methods section. cDNA codon numbers are displayed in the electropherograms. The site of mutation is indicated by an arrow. (I) The insertion of cytosine between codons 587 and 588 in exon 3 leads to a frame-shift with introduction of a stop codon at position 205 of the $\alpha \mathrm{ENaC}$ protein (p.Pro197Alafs ${ }^{*}$ ). The consanguineous parents and the two healthy brothers are heterozygotes. (II) Insertion of TACA between codons 1342 and 1343 results in a frame-shift at residue 448 and introduces a stop codon at position 460 (p.Arg448llefs ${ }^{*} 13$ ). The parents are heterozygous carriers. A sister carries the wild type sequence on both alleles. (III) A deletion of guanine at residue 742 in exon 4 introduces a premature stop codon at position 248 of the $\alpha \mathrm{ENaC}$ protein $($ Val248*). The parents are heterozygous for this mutation, the siblings were not investigated. (IV) The patient carries the same mutation as patient 1 (p.Pro197Alafs ${ }^{*}$, panel I). The parents are the carriers. The patient's siblings were not tested. (V) Based on a change from C to T at position 1474 in exon 10, arginine at position 492 is replaced by a stop codon (Arg492*). The parents and a sister are heterozygous for the Arg $492^{*}$ mutation. A brother who died in the neonatal period was not analyzed. Noteworthy is the frequency of infantile death in the consanguineous family. (VI) Cytosine at position 189 of exon 2 is substituted by adenine, resulting in an amino acid exchange from cysteine to stop at position 63 of the mature protein $\left(\mathrm{Cys}^{*} 3^{*}\right.$. The consanguineous parents are heterozygous for this mutation. (VII) Intron-exon boundary of intron 8 /exon 9 . The start of exon 9 is highlighted by an asterisk. Adenine is replaced by guanine, changing the splice acceptor site from $A G$ to $G G$ (c.1361-2A $>$ G). The consanguineous parents are asymptomatic carriers. Siblings were not tested so far. 
the first postnatal days and first noted when patients exhibit signs of dehydration. Patients seem to be able to adapt to electrolyte imbalances and tolerate potassium levels way above the physiologic range without cardiac impairment. In our patients, mean sodium levels at presentation were low $(117.6 \mathrm{mmol} / \mathrm{l}$; range 109-127), and potassium levels were two times above the physiologic upper range $(10.6 \mathrm{mmol} / \mathrm{l}$; range 8.4-13.0). Therefore, all the patients required intensive care and different therapeutic approaches to lower life-threatening hyperkalemia. In some patients, i.v. sodium supplementation and the application of albuterol or glucose/insulin were sufficient to re-establish $\mathrm{Na} / \mathrm{K}$ balance. Some patients, however, additionally needed ion exchange resins or even dialysis. The typical constellation of laboratory values, comprising hyperkalemia, hyponatremia, acidosis, elevated urinary sodium excretion, highly elevated aldosterone levels, and elevated sweat or salivary sodium led to the diagnosis of PHA1. The fact that all patients were born to consanguineous couples further narrowed down the differential considerations to systemic PHA1. Consequently, we did not analyze the NR3C2 gene, being the cause of autosomal dominant PHA1.
All our patients had underlying homozygous variations in the SCNN1A gene. None of them was found in the SNPper database (21). Thus, based on the clinical phenotype we regard them as pathogenic although we did not perform functional in vitro studies. However, we think that conclusions can be drawn by analogy to proven mutations.

The $\alpha$ subunit is obligatorily required for channel activity, while the $\beta$ and $\gamma$ subunits are necessary for channel expression and activity at the cell surface (22). More than half of the published mutations in systemic PHA1 were detected in the SCNN1A gene. Patient 5 carries a SCNN1A mutation that had been previously described (23) with the difference that we denoted, the mutation c. $1474 \mathrm{C}>\mathrm{T}$ instead of $1473 \mathrm{C}>\mathrm{T}$ was based on the cDNA reference sequence (NM_001038.5). The residual activity of the resulting Arg492* mutant protein is $2 \%(23)$, thereby explaining the patient's phenotype. All other individuals carried novel stop or frame-shift mutations truncating the $\alpha \mathrm{ENaC}$ subunit proteins. The $\mathrm{C} 63 \mathrm{X}$ mutation leads to an $\alpha \mathrm{ENaC}$ subunit protein lacking both membrane spanning domains and the extracellular loop (11). Two other mutations with comparable impact on the protein
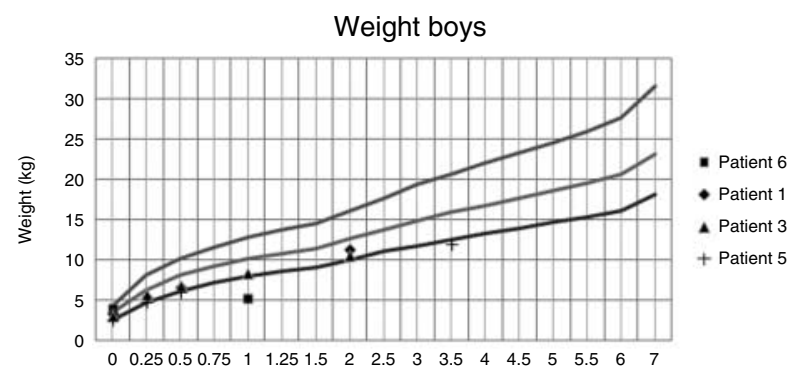

Height boys

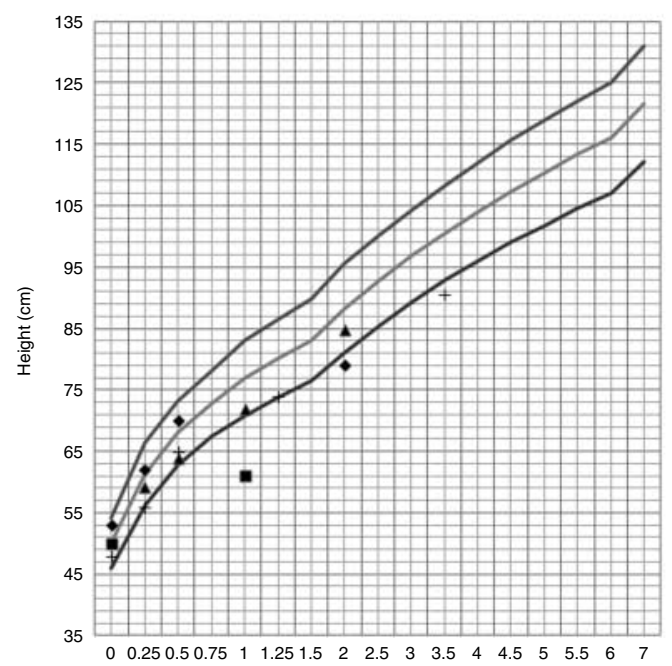

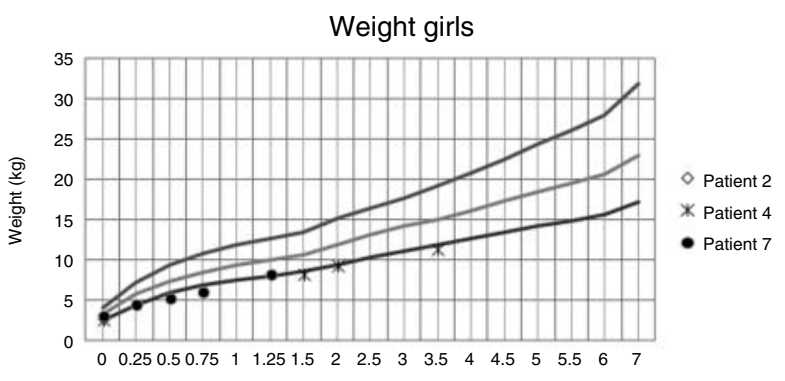

Height girls

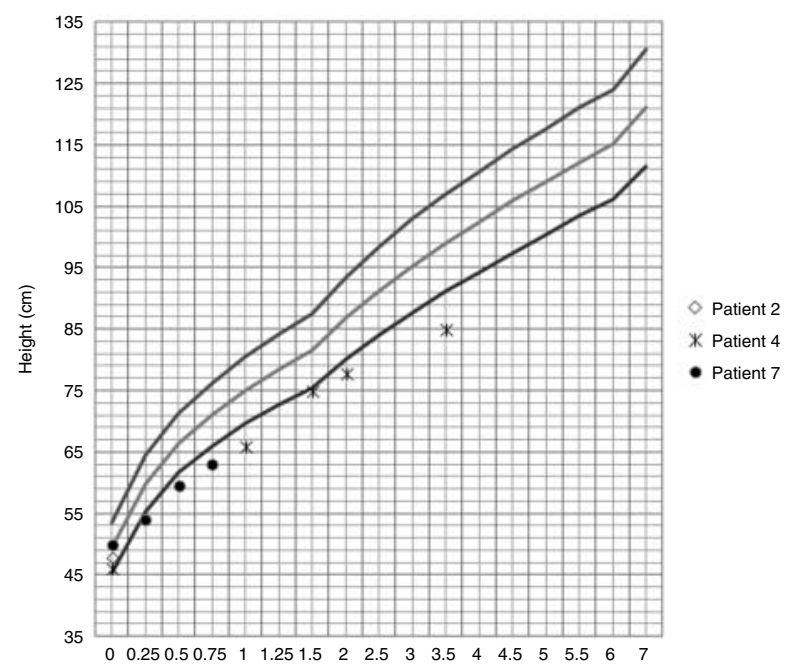

Figure 2 Growth charts showing the 3rd, 50th, and 97th centile for height (lower panel) and weight (upper panel) from birth to the age of 7 years old according to updated Turkish normative data (18). Patients' data are plotted. Almost all patients struggle to gain weight during the first year of life. Height velocity is in the lower range or even decreased. 
structure, R56X and I68fs, have been published earlier $(4,6)$. The I68fs mutation, if co-expressed with the beta and gamma subunits in Xenopus laevis oocytes, completely abolishes sodium currents (24). Based on the clinical phenotype, the Cys63* mutation is likely to have a comparable impact on $\mathrm{ENaC}$ function. The mutations Pro197Alafs*9, Arg448Ilefs*13, and Val248* introduce premature stop codons deleting the pore-forming region and the intracellular C-terminus. A deleted or malfunctioning pore-forming region is also likely resulting from the intron 8 splice-site mutation. Previous studies suggest that all stop mutations within the extracellular loop affect $\mathrm{ENaC}$ function alike (23, 25, 26). Only missense mutations in the SCNN1A were found to result in retained channel function $(12,27,28)$.

Six out of seven patients studied are of Turkish origin, suggesting that $S C N N 1 A$ mutations are the most frequent, especially in Turkey. However, this could be a selection bias due to the collaboration among the centers as other groups did not corroborate this $(29,30,31)$. Two of our patients even carried the same mutation. As both families live in the middle part of Germany they might be related to each other without knowing it.

Patients with systemic PHA1 required life-long medical assistance and follow-up. The primary aim is to keep sodium and potassium levels within the normal range, which in daily practice may be hard to achieve. So far, there are no evidence-based recommendations as to how to treat PHA1. The dosage of sodium per day to establish salt homeostasis varies greatly and may be as high as ten times the physiologic need. While rather low sodium doses can be administered orally using $\mathrm{NaHCO} 3-, \mathrm{NaCl}$, or Na-citrate, some children need nasogastric tubes or enterostomies because they are reluctant to swallow the salty fluids or pills. Especially, during infancy sodium supplementation has to be readjusted frequently due to changes in body weight. Therefore, most of the patients reported here were followed-up every 8-12 weeks during the first years. Moreover, salt-loss during acute illnesses can be life-threatening (as can be seen in patients 5 and 6). Thus, parents need to be instructed accordingly.

Potassium levels are not sufficiently influenced by sodium supplementation. All of the patients reported here were put on ion-exchange resins administered orally. A dose of $1-2 \mathrm{~g} / \mathrm{kg}$ body weight of resonium was sufficient to control potassium levels in most of the patients.

The atopic dermatitis-like rash can give a clinical hint toward systemic PHA1. It is considered to be the result of increased salt-loss through the skin, which causes inflammation in the eccrine structures $(32,33)$. However, as can be seen in our cohort, only three out seven patients exhibited such symptoms (patient 3, 5, and 6). As two of the patients also had recurrent salt-losing crises, for the clinician it might also be a warning sign to follow the patient more closely.

The growth charts (Fig. 2) show that in the majority of cases, patients thrive poorly during the first 2 years. This is also the most critical period of salt-losing crises. Despite all the complications in early infancy, all the six patients alive had a normal psycho-motor development, a fact that could be used to encourage parents to adhere to best therapy, which is essential in systemic PHA1.

The pulmonary symptoms that occur in many patients are the result of an impaired ion current through the epithelium resulting in airway obstruction by over-secreted alveolar fluid $(4,34,35)$. While ENaCdeficient mice die in early life (34), human patients were reported to improve over the time, possibly due to growth of the airways (4). Why some patients exhibit more symptoms than others seems to be influenced by individual factors rather than by the underlying mutation. Of the seven patients described in this paper, five displayed pulmonary symptoms, independent of the length of the truncated protein. This is in accordance with data published by Kerem et al. (4). Local differences could be studied systematically measuring nasal potential differences (PD). However, our cohort comprised seven patients from three different countries, treated in seven different centers. Therefore, it is impossible to measure nasal PD systematically in our patients. Four patients had recurrent infections and one had bronchiectasis, which is rather uncommon in systemic PHA1. As the patients with recurrent infections are $<3$ years old, we do not know whether their pulmonary symptoms may worsen or improve over the years.

In conclusion, our data on the novel SCNN1A mutations and clinical phenotypes add further information on mutations in the ENaC. We corroborate previous studies showing that our current knowledge on systemic PHA1 is too limited to explain or even to predict the clinical phenotype, in particular the pulmonary phenotype. Systematic studies on the local expression of proteins involved in $\mathrm{ENaC}$ trafficking in PHA1 patients could further enrich our understanding of PHA1 pathophysiology.

\section{Supplementary data}

This is linked to the online version of the paper at http://dx.doi.org/ 10.1530/EJE-12-1000.

\section{Declaration of interest}

The authors declare that there is no conflict of interest that could be perceived as prejudicing the impartiality of the research reported.

\section{Funding}

This research did not receive any specific grant from any funding agency in the public, commercial, or not-for-profit sector. 


\section{References}

1 Geller DS, Rodriguez-Soriano J, Vallo Boado A, Schifter S, Bayer M, Chang SS \& Lifton RP. Mutations in the mineralocorticoid receptor gene cause autosomal dominant pseudohypoaldosteronism type I. Nature Genetics 199819 279-281. (doi:10.1038/966)

2 Riepe FG. Clinical and molecular features of type 1 pseudohypoaldosteronism. Hormone Research 200972 1-9. (doi:10.1159/ 000224334 )

3 Hubert EL, Teissier R, Fernandes-Rosa FL, Fay M, Rafestin-Oblin ME, Jeunemaitre X, Metz C, Escoubet B \& Zennaro MC. Mineralocorticoid receptor mutations and a severe recessive pseudohypoaldosteronism type 1. Journal of the American Society of Nephrology 201122 1997-2003. (doi:10.1681/ASN.2011030245)

4 Kerem E, Bistritzer T, Hanukoglu A, Hofmann T, Zhou Z, Bennett W, MacLaughlin E, Barker P, Nash M, Quittell L et al. Pulmonary epithelial sodium-channel dysfunction and excess airway liquid in pseudohypoaldosteronism. New England Journal of Medicine $1999 \mathbf{3 4 1}$ 156-162. (doi:10.1056/NEJM19990 7153410304)

5 Dirlewanger M, Huser D, Zennaro MC, Girardin E, Schild L \& Schwitzgebel VM. A homozygous missense mutation in SCNN1A is responsible for a transient neonatal form of pseudohypoaldosteronism type 1. American Journal of Physiology. Endocrinology and Metabolism 2011301 E467-E473. (doi:10.1152/ajpendo. 00066.2011)

6 Chang SS, Grunder S, Hanukoglu A, Rosler A, Mathew PM, Hanukoglu I, Schild L, Lu Y, Shimkets RA, Nelson-Williams C et al. Mutations in subunits of the epithelial sodium channel cause salt wasting with hyperkalaemic acidosis, pseudohypoaldosteronism type 1. Nature Genetics 199612 248-253. (doi:10.1038/ ng0396-248)

7 Canessa CM, Schild L, Buell G, Thorens B, Gautschi I, Horisberger JD \& Rossier BC. Amiloride-sensitive epithelial $\mathrm{Na}^{+}$ channel is made of three homologous subunits. Nature 1994367 463-467. (doi:10.1038/367463a0)

8 Butterworth MB, Weisz OA \& Johnson JP. Some assembly required: putting the epithelial sodium channel together. Journal of Biological Chemistry $2008 \mathbf{2 8 3}$ 35305-35309. (doi:10.1074/ jbc.R800044200)

9 Jasti J, Furukawa H, Gonzales EB \& Gouaux E. Structure of acidsensing ion channel 1 at 1.9 A resolution and low $\mathrm{pH}$. Nature 2007449 316-323. (doi:10.1038/nature06163)

10 Stockand JD, Staruschenko A, Pochynyuk O, Booth RE \& Silverthorn DU. Insight toward epithelial $\mathrm{Na}^{+}$channel mechanism revealed by the acid-sensing ion channel 1 structure. IUBMB Life $2008 \mathbf{6 0}$ 620-628. (doi:10.1002/iub.89)

11 Canessa CM, Merillat AM \& Rossier BC. Membrane topology of the epithelial sodium channel in intact cells. American Journal of Physiology 1994267 C1682-C1690.

12 Grunder S, Firsov D, Chang SS, Jaeger NF, Gautschi I, Schild L, Lifton RP \& Rossier BC. A mutation causing pseudohypoaldosteronism type 1 identifies a conserved glycine that is involved in the gating of the epithelial sodium channel. EMBO Journal 1997 16 899-907. (doi:10.1093/emboj/16.5.899)

13 Kellenberger S, Gautschi I \& Schild L. A single point mutation in the pore region of the epithelial $\mathrm{Na}^{+}$channel changes ion selectivity by modifying molecular sieving. PNAS 199996 4170-4175. (doi:10.1073/pnas.96.7.4170)

14 Riepe FG, van Bemmelen MX, Cachat F, Plendl H, Gautschi I, Krone N, Holterhus PM, Theintz G \& Schild L. Revealing a subclinical salt-losing phenotype in heterozygous carriers of the novel S562P mutation in the $\alpha$ subunit of the epithelial sodium channel. Clinical Endocrinology 200970 252-258. (doi:10.1111/ j.1365-2265.2008.03314.x)

15 Shimkets RA, Warnock DG, Bositis CM, Nelson-Williams C, Hansson JH, Schambelan M, Gill JR Jr, Ulick S, Milora RV, Findling JW et al. Liddle's syndrome: heritable human hypertension caused by mutations in the beta subunit of the epithelial sodium channel. Cell 199479 407-414. (doi:10.1016/0092-8674(94) 90250-X)

16 Cole TJ, Freeman JV \& Preece MA. British 1990 growth reference centiles for weight, height, body mass index and head circumference fitted by maximum penalized likelihood. Statistics in Medicine 199817 407-429. (doi:10.1002/(SICI)1097-0258(19980228) $17: 4<407::$ AID-SIM742>3.0.CO;2-L)

17 Brandt I \& Reinken L. The growth rate of healthy children in the first 16 years: Bonn-Dortmund longitudinal developmental study. Klinische Padiatrie 1988200 451-456. (doi:10.1055/s-20081033752)

18 Gokcay G, Furman A \& Neyzi O. Updated growth curves for Turkish children aged 15 days to 60 months. Child: Care, Health and Development 200834 454-463. (doi:10.1111/j.1365-2214. 2008.00813.x)

19 den Dunnen JT \& Antonarakis SE. Nomenclature for the description of human sequence variations. Human Genetics 2001 109 121-124. (doi:10.1007/s004390100505)

20 Martinerie L, Pussard E, Foix-L'Helias L, Petit F, Cosson C, Boileau P \& Lombes M. Physiological partial aldosterone resistance in human newborns. Pediatric Research 200966 323-328. (doi:10.1203/PDR.0b013e3181b1bbec)

21 Riva A \& Kohane IS. A SNP-centric database for the investigation of the human genome. BMC Bioinformatics 2004533. (doi:10.1186/1471-2105-5-33)

22 Schild L. The epithelial sodium channel and the control of sodium balance. Biochimica et Biophysica Acta $2010 \mathbf{1 8 0 2} 1159-1165$. (doi:10.1016/j.bbadis.2010.06.014)

23 Bonny O, Knoers N, Monnens L \& Rossier BC. A novel mutation of the epithelial $\mathrm{Na}^{+}$channel causes type 1 pseudohypoaldosteronism. Pediatric Nephrology 200217 804-808. (doi:10.1007/ s00467-002-0945-8)

24 Bonny O \& Rossier BC. Disturbances of Na/K balance: pseudohypoaldosteronism revisited. Journal of the American Society of Nephrology 200213 2399-2414. (doi:10.1097/01.ASN.00000 28641.59030.B2)

25 Bonny O, Chraibi A, Loffing J, Jaeger NF, Grunder S, Horisberger JD \& Rossier BC. Functional expression of a pseudohypoaldosteronism type I mutated epithelial $\mathrm{Na}^{+}$channel lacking the pore-forming region of its $\alpha$ subunit. Journal of Clinical Investigation $1999 \mathbf{1 0 4}$ 967-974. (doi:10.1172/JCI6821)

26 Schaedel C, Marthinsen L, Kristoffersson AC, Kornfalt R, Nilsson KO, Orlenius B \& Holmberg L. Lung symptoms in pseudohypoaldosteronism type 1 are associated with deficiency of the $\alpha$-subunit of the epithelial sodium channel. Journal of Pediatrics 1999135 739-745. (doi:10.1016/S0022-3476(99) 70094-6)

27 Edelheit O, Hanukoglu I, Gizewska M, Kandemir N, TenenbaumRakover Y, Yurdakok M, Zajaczek S \& Hanukoglu A. Novel mutations in epithelial sodium channel (ENaC) subunit genes and phenotypic expression of multisystem pseudohypoaldosteronism. Clinical Endocrinology $2005 \mathbf{6 2}$ 547-553. (doi:10.1111/j.13652265.2005.02255.x)

28 Firsov D, Robert-Nicoud M, Gruender S, Schild L \& Rossier BC. Mutational analysis of cysteine-rich domains of the epithelium sodium channel $(\mathrm{ENaC})$. Identification of cysteines essential for channel expression at the cell surface. Journal of Biological Chemistry $19992742743-2749$. (doi:10.1074/jbc.274.5.2743)

29 Hanukoglu A. Type I pseudohypoaldosteronism includes two clinically and genetically distinct entities with either renal or multiple target organ defects. Journal of Clinical Endocrinology and Metabolism 199173 936-944. (doi:10.1210/jcem73-5-936)

30 Hanukoglu A, Bistritzer T, Rakover Y \& Mandelberg A. Pseudohypoaldosteronism with increased sweat and saliva electrolyte values and frequent lower respiratory tract infections mimicking cystic fibrosis. Journal of Pediatrics 1994125 752-755. (doi:10.1016/ S0022-3476(06)80176-9) 
31 Zennaro MC, Hubert EL \& Fernandes-Rosa FL. Aldosterone resistance: structural and functional considerations and new perspectives. Molecular and Cellular Endocrinology $20123 \mathbf{3 5 0}$ 206-215. (doi:10.1016/j.mce.2011.04.023)

32 Martin JM, Calduch L, Monteagudo C, Alonso V, Garcia L \& Jorda E. Clinico-pathological analysis of the cutaneous lesions of a patient with type I pseudohypoaldosteronism. Journal of the European Academy of Dermatology and Venereology 200519 377-379. (doi:10.1111/j.1468-3083.2004.01173.x)

33 Urbatsch A \& Paller AS. Pustular miliaria rubra: a specific cutaneous finding of type I pseudohypoaldosteronism. Pediatric Dermatology 200219 317-319. (doi:10.1046/j.1525-1470.2002.00090.x)

34 Hummler E, Barker P, Gatzy J, Beermann F, Verdumo C, Schmidt A, Boucher R \& Rossier BC. Early death due to defective neonatal lung liquid clearance in $\alpha$-ENaC-deficient mice. Nature Genetics 1996 12 325-328. (doi:10.1038/ng0396-325)
35 Hummler E \& Planes C. Importance of ENaC-mediated sodium transport in alveolar fluid clearance using genetically-engineered mice. Cellular Physiology and Biochemistry $2010 \quad 25$ 63-70. (doi:10.1159/000272051)

36 Bennett RL, French KS, Resta RG \& Doyle DL. Standardized human pedigree nomenclature: update and assessment of the recommendations of the National Society of Genetic Counselors. Journal of Genetic Counseling 200817 424-433. (doi:10.1007/s10897008-9169-9)

Received 14 November 2012

Revised version received 23 January 2013

Accepted 12 February 2013 